\title{
Neutron noise analysis of simulated mechanical and thermal-hydraulic perturbations in a PWR core
}

\author{
L.A. Torres ${ }^{\text {a }}$, D. Chionis ${ }^{b}$, C. Montalvo ${ }^{\mathrm{a}, *}$, A. Dokhane ${ }^{\mathrm{b}}$, A. García-Berrocal ${ }^{\mathrm{a}}$ \\ a Energy and Fuels Department, Technical University of Madrid (UPM), Ríos Rosas 21, 28003 Madrid, Spain \\ ${ }^{\mathrm{b}}$ Paul Scherrer Institut, Laboratory for Reactor Physics and Thermal-Hydraulics, Forschungsstrasse 111, 5232 Villigen-PSI, Switzerland
}

\section{A R T I C L E I N F O}

\section{Article history:}

Received 12 September 2018

Received in revised form 12 November 2018

Accepted 14 November 2018

\section{Keywords:}

Noise analysis

Neutron noise

SIMULATE-3K

Mechanical vibrations

Thermal-hydraulic perturbations

\begin{abstract}
A B S T R A C T
KWU pre-Konvoi PWRs (SIEMENS design) are commonly exhibiting high neutron noise levels which can lead to costly operational issues (i.e. activation of SCRAM system, operation of unrated core power, etc.). The frequency region of interest of neutron noise is below $1 \mathrm{~Hz}$, which is the typical frequency range of thermal-hydraulic phenomena. This feature seems to indicate that, coolant flow and temperature oscillations can have a key impact on neutron noise phenomena. Moreover, an increasing neutron noise trend (in term of normalized root mean square) was recently observed in many KWU-PWRs. This increasing trend has been speculated to be correlated with the introduction of a new fuel design type in the KWU-PWRs. This fact indicates that there should be some correlation between neutron noise spectral characteristics and fuel assemblies' performance. In order to advance in understanding this phenomenon, the transient nodal code SIMULATE-3K (S3K) has been used to simulate mechanical vibrations of fuel assemblies and thermal-hydraulic fluctuations of the core inlet flow and temperature. The simulated neutron detectors responses are analysed with noise analysis techniques and compared to real plant data. This analysis indicates that the cross-feedback between the mechanical and thermal-hydraulic disturbances complicate the identification of the origin of the perturbation source. The simulated results indicate that the neutron noise spectral characteristics can be associated separately to different causes. In this sense, the results of this work seem to indicate that the spectral features of the neutron noise are a consequence of both mechanical perturbations and thermal-hydraulic fluctuations.
\end{abstract}

(c) 2018 Elsevier Ltd. All rights reserved.

\section{Introduction}

In a region of the reactor, neutron noise can be defined as to the series of neutron flux fluctuations around the mean value which is considered stationary in a certain time period (Hashemian, 2006). Neutron noise carries information about the processes that are taking place in the reactor (Dykin et al., 2014; Pázsit et al., 2014; Pázsit et al., 2010; Runkel, 1987; Thie, 1981). These fluctuations are detected by a series of in-core and ex-core sensors that are part of the reactor instrumentation system.

The neutron noise phenomenon occurs in all types of nuclear reactors. In the particular case of KWU pre-Konvoi pressurized water reactors (KWU-PWRs), high values of neutron noise have been observed since the beginning of their operation (Kostic et al., 1989; Kostic et al., 1990; Kostic, 1991; Laggiard and Runkel, 1997; Runkel, 1987; Stegemann and Runkel, 1996). Even if the neutron noise phenomena do not correspond to any safety related problems, these reactors have to actively take various mea-

\footnotetext{
* Corresponding author.
}

sures in order to mitigate the high neutron noise amplitudes. In the past, many KWU-PWRs installed signal filters at the first cycles of operation in order to avoid the unnecessary activation of the SCRAM system whenever the neutron noise levels were exceeding a certain threshold (Grondey et al., 1991). In some cases, KWUPWRs had to perform automatic power reductions during the first years of operation for preventing the neutron noise levels to reach higher values (Grondey et al., 1991; Stegemann and Runkel, 1996). All these measures were clearly affecting the efficient and economical operation of the reactor, and consequently, neutron noise was leading to costly operational issues for the utilities.

In typical KWU-PWRs the low frequency range (below $1 \mathrm{~Hz}$ ) of neutron noise contains more than $95 \%$ of the total signal, and it decreases exponentially with higher frequencies (Seidl et al., 2015). This spectral region has been studied for surveillance purposes and for detecting core flow anomalies in several papers (Bermejo et al., 2014; Kostic et al., 1989; Kostic et al., 1990; Rubiolo and Young, 2008). This low spectral range coincides with the frequency of maximum response due to thermal-hydraulic parameters fluctuations, the heat transfer processes, as well as 
the moderator temperature coefficient (MTC) feedback (Fry et al., 1984; Runkel, 1987; Thie, 1981; Zylbersztejn et al., 2013). The relationship of neutron noise behaviour and thermal-hydraulic oscillations has been addressed in several studies, where simulations of scenarios that consider flow and temperature oscillations have been analysed (Bermejo et al., 2017; Chionis et al., 2017; Chionis et al., 2018).

However, recently there has been an increase in the neutron noise normalized root mean square (NRMS) that coincides over time with the introduction of a new type of fuel elements, leading again to automatic power reductions (RSK/ESK, 2013; Spanish Nuclear Safety Council, 2011). This fact points out to the fuel elements as a possible direct cause on the neutron noise increasing trend (Tran et al., 2015). In (Viebach et al., 2018), the authors showed correlations between the increased neutron noise level and the change in the fuel elements eigenfrequency. In this sense, the interest arises to study simulations where mechanical vibrations of fuel elements are considered.

In order to understand the neutron noise phenomenon, the transient nodal code SIMULATE-3K (S3K) is used to simulate certain stochastic perturbation scenarios. On the one hand, lateral vibrations of fuel assemblies are simulated, and on the other hand, thermal-hydraulic parameters fluctuations are imposed to occur at the core inlet. The simulation results are analysed with noise analysis techniques and compared to KWU-PWR plant data.

This paper is structured as follows: First, the neutron noise phenomenon and its characteristics are introduced in Section 2. Then, the neutron noise analysis techniques which have been utilized in this work are presented in Section 3, followed by the description of the simulation methodology with S3K in Section 4. The analysed simulated scenarios are briefly presented in Section 5. Section 6 and 0 assess extensively the simulated neutron noise characteristics by comparing against real plant data observations. Finally, concluding remarks are given in Section 8.

\section{Neutron noise phenomenon}

The neutron flux density at any region of the core, as recorded by neutron detectors, it is expected to have a constant value over time under steady-state operating conditions. Nevertheless, very small fluctuations around the signal mean value can be recorded if the utilized measuring techniques are sufficiently sensitive enough. These fluctuations are commonly referred as signal noise. Noise is also observed in various control parameters such as pressure, flow, temperature and mechanical vibrations.

The neutron noise can be detected by a series of sensors located inside and outside the core; namely the in-core and ex-core neutron detectors. Signal analysis techniques already demonstrate that the neutron noise signals are correlated in both time and space, at a certain extent, even if they have a stochastic nature.

It is known that the changes in the mean value in the reactor neutronics are linked to the evolution of thermal-hydraulic and mechanical processes, however, the cross and simultaneous feedbacks among these three types of processes make the identification of their relationships a quite complex problem, and consequently, hard to quantify (Pázsit et al., 2016; Runkel, 1987). A certain level of correlation is observed among the different processes that transfer these random fluctuations throughout the reactor, i.e., mechanical vibrations and thermal-hydraulic oscillations.

\section{Noise analysis techniques}

The results and conclusions obtained in this study are based on the use of analysis tools set, the so-called noise analysis techniques, that have been used for various purposes in nuclear reactor applica- tions (Czibók et al., 2003; Hashemian, 2011a; Ortiz-Villafuerte et al., 2006). Sensor surveillance (Hashemian, 2011b; Hashemian and Jiang, 2010; Montalvo et al., 2012; Montalvo et al., 2014a,b) and neutron noise analysis for core diagnostics (Montalvo et al., 2016; Pázsit et al., 2016) are two of the main applications of noise analysis techniques. Besides recently, and due to the increase in neutron noise levels in certain German, Spanish and Swiss nuclear reactors, these techniques are being used to comprehend the results from certain simulations (Bermejo et al., 2017; Chionis et al., 2017; Viebach et al., 2018). Therefore, noise analysis techniques are very beneficial for the comparison/validation of simulated results against plant measurements.

The main tool of noise analysis techniques is based on observing the response of the neutron detector sensors in the frequency domain through the Fourier transform of the autocorrelation function (Newland, 2012), the so-called auto power spectral density (APSD) defined as:

$\operatorname{APSD}(f)=\int_{-\infty}^{\infty} C_{x x}(\tau) e^{-j 2 \pi f \tau} d \tau$

being $\tau$ the lag used when estimating the autocorrelation function of the sensor output signal $C_{x x}(\tau)$ which is defined as:

$C_{x x}(\tau)=E[x(\tau) x(t+\tau)]$

being $E$ the expected value and $x(t)$ the sensor output signal. The autocorrelation function can be estimated by considering that the sensor output signal is a discrete time series $x_{i}$ with $N$ points record length which can be lagged $k$ times:

$C_{k}=\frac{1}{N-k} \sum_{i=1}^{N-k} x_{i} x_{i+k} k=1,2 \ldots N-1$

Analogously, for two variables, we can calculate the cross power spectrum density (CPSD) through the Fourier transform of the cross correlation between both signals:

$\operatorname{CPSD}(f)=\int_{-\infty}^{\infty} C_{x y}(\tau) e^{-j 2 \pi f \tau} d \tau$

being $C_{x y}$ the cross correlation between signal $x$ and $y$, defined as:

$C_{x y}(\tau)=E[x(t) y(t+\tau)]$

These functions can be used to calculate the signals' pairwise coherence ( $\mathrm{COH} 2)$ and their phase difference (PHASE), defined as:

$\mathrm{COH}^{2}(f)=\frac{|C P S D(f)|^{2}}{\operatorname{APSD}_{1}(f) \operatorname{APSD}_{2}(f)}$

$\operatorname{PHASE}(f)=\operatorname{arctg} \frac{\operatorname{Im}|C P S D(f)|}{\operatorname{Re}|C P S D(f)|}$

The coherence takes values between 0 and 1 . A zero coherence $\left(\mathrm{COH}^{2} \approx 0\right)$ for some considered frequency means that the two analyzed signals' contents are not correlated, or its correlation is very low. A value $\mathrm{COH}^{2} \approx 1$ means that the two considered signals' contents are highly correlated. The PHASE is defined as the phase angle difference between the analysed pair of signals.

However, the estimated APSDs based on plant data do not have always a smooth behavior, and it is preferable to fit the APSDs to a nonlinear function in order to estimate more accurately certain parameters, such as the response time, eigenfrequencies, amplitudes, damping, etc. Consequently, an autoregressive (AR) model is commonly used to this purpose (Blázquez and Ballestrín, 1995; Montalvo et al., 2012). The AR coefficients $a_{k}$ are derived by solving the Yule Walker equation:

$C_{j}=\sum_{k=1}^{n} a_{k} \cdot C_{j-k} \quad j=0 \ldots N$ 
being $n$ the AR model order and $C_{j}$ the values of the estimated correlation function at the different $j$ lags. The expression for the AR model is expressed as:

$x_{i}=\sum_{k=1}^{n} x_{i-k} a_{k}+\varepsilon_{i} i=0 \ldots N$

being $a_{k}$ the autoregressive coefficients of the AR model, $\varepsilon_{i}$ the input driven white noise, and $x i$ the output sensor signal. The AR model order can be inferred with the Akaike Information Criterion (AIC) (Akaike, 1974).

\section{Preparation of simulated scenarios}

Neutron noise is a rather complex phenomenon depending on various stochastic processes taking place inherently in any nuclear reactor. These stochastic processes can be related, among others, to fluctuation of thermal-hydraulic parameters (e.g. inlet coolant temperature and flow, etc.) or to vibration of fuel assemblies and structural materials. The modelling of these stochastic processes with advanced full core simulators is beneficial for the systematic analysis of the neutron noise characteristics. In this paper, the PWR core developed in the framework of the OECD/NEA transient benchmark (Kozlowski and Downar, 2003) has been used to simulate stochastic perturbations and to assess their impact on neutron noise phenomenology. This core has been modelled using the CASMO-5/SIMULATE-3 code sequence and the dynamic simulations have been performed through the S3K code. S3K is a transient nodal simulator including coupled neutronic and thermalhydraulic capabilities.

\subsection{Thermal-hydraulic parameters fluctuations}

In the framework of this project, the inlet coolant flow and temperature are modelled to fluctuate randomly over time using the S3K code. The user can impose time-dependent fluctuations of these parameters using the input cards HYD.CLG, HYD.CLT, and HYD.CLW, as already presented in (Chionis et al., 2018). The analysed core is modelled to have four inlet coolant loops, as presented in Fig. 2. In a simplified approach it is assumed that a fluctuation which is taking place in one coolant loop will mainly affect the inlet conditions of those fuel elements which are azimuthally closer to that specific loop. The dependency of the fuel assemblies inlet conditions to the inlet coolant fluctuations is defined by the user by utilizing respective weighted factors (HYD.CLW input card in S3K). The utilized weighted factors are presented in Fig. 1. As an example, the inlet coolant conditions of the fuel assemblies located at the south-west quarter-core are simulated to be mainly affected (weighted factor equal to 1 ) by the coolant fluctuations of inlet coolant loop 1, and so on and so forth.

In addition, the inlet coolant conditions can be fluctuated either in a synchronized or in an unsynchronized manner. In case of synchronized fluctuation, all the four coolant loops are following the exact same random change of the inlet coolant conditions (temperature and/or flow), between each other, during a time step $t$. In the next time step $t+1$, the inlet coolant conditions will be modified to a new random value. This modification will be applied equally to all four coolant loops. This procedure is followed for every time step of the simulation. In case of unsynchronized perturbation, the inlet coolant conditions are different (uncorrelated) between all the four coolant loops during a time step $t$. Therefore, a modification of the inlet coolant conditions in one coolant loop will be unsynchronized compared to all the other coolant loops. This procedure is followed during all time steps of the simulation. Moreover, with the help of a script written in MATLAB, it is possible to automatize the preparation of the input deck which describes the time-dependent fluctuation of the thermal-hydraulic parameters of interest. With the help of this in-house script, the user can select which parameters are fluctuated, the amplitude of fluctuation, and the dependency between the inlet loops (synchronized or not).

\subsection{Fuel assembly vibrations}

One of the latest version of S3K (i.e. S3K-v2.06.00) offers the capability to mimic the lateral vibration of fuel assemblies in a time-dependent way. This can be achieved by modifying the water gap widths between a fuel assembly which is selected to vibrate and its neighbouring assemblies. The dynamic modification of the water gaps has a direct impact on the time-dependent variation of the homogenized nodal two-group cross-sections allowing the user to model the lateral movement in the $\mathrm{x}$ - and/or $\mathrm{y}$ direction. It is to note, that the capability of S3K to properly simulate fuel assemblies vibrations has been already presented in (Chionis et al., 2017). The fuel assembly model in S3K is activated by the KIN.BOW input card. In addition, a set of MATLAB scripts has been developed by PSI in order to automatize the generation of the input file which describes the time-dependent variation of the water gap widths of the vibrating fuel assemblies. With the help of this complementary set of scripts, the user can control the number and the exact locations of the vibrating fuel assemblies, the amplitude of lateral displacement, the direction of movements (in the $\mathrm{x}$ - and/or the $\mathrm{y}$-direction), the type of perturbation (i.e. random over time, or following a sine wave function with a specific nominal frequency), and the dependency of movement between the vibrating fuel assemblies (i.e. identical movement between
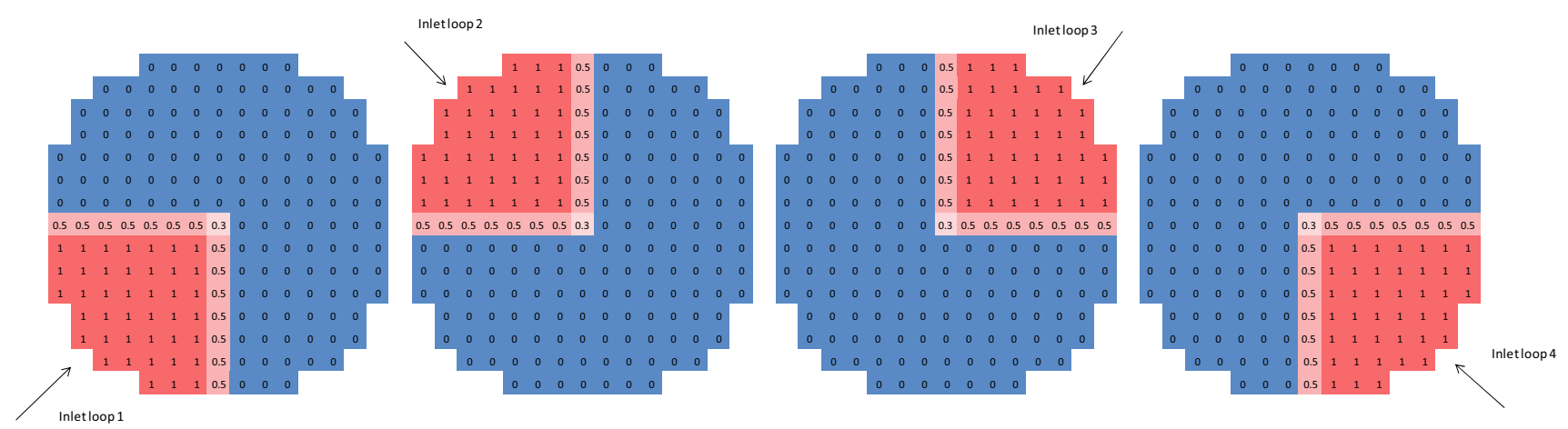

Fig. 1. Dependency of fuel assemblies' inlet coolant conditions on the coolant fluctuation taking place in the four coolant inlet loops. 
all the vibrating fuel assemblies; synchronized vibration, or uncorrelated movement; unsynchronized vibration).

\section{Analysed scenarios}

This section describes the various analysed scenarios simulated with S3K. The outputs of these simulations are the responses from in-core and ex-core neutron detectors. All the scenarios' output include 8 signals from the ex-core detectors placed at 4 different radial locations and at 2 axial levels, and 48 signals from in-core detectors placed at 8 different radial positions and at 6 axial levels. Fig. 2 shows the core layout with the locations of both the in-core (i.e. 05, N12, J2, J6, G10, G14, C4 and B11) and the ex-core detectors (i.e. S1, S2, S3 and S4). The axial levels are listed from the lower to the higher part, that is, from axial level 1 ( $\operatorname{Lv} 1)$ to axial level 6 (Lv 6).

The scenarios are divided in two categories; on the one hand, scenarios in which mechanical vibrations of the fuel elements are analysed, and on the other hand, scenarios in which thermalhydraulic oscillations are simulated.

The mechanical vibrations scenarios will be referred as follows:

a.1: Synchronized vibration of the central cluster of $5 \times 5$ fuel assemblies only in the $x$-direction following a white noise signal (i.e. random displacement) with a maximum displacement amplitude of $1.1 \mathrm{~mm}$ (Fig. 2, left plot).

a.2: Vibration of a single fuel assembly, located at the position $\mathrm{E} 4$, only in the $\mathrm{X}$-direction following a white noise signal (i.e. random displacement) with a maximum displacement amplitude of $1.1 \mathrm{~mm}$ (Fig. 2, right plot).

The thermal-hydraulic oscillations scenarios will be referred as follows:

b.1 Synchronized fluctuation of inlet coolant temperature between all the four coolant loops. The inlet coolant temperature is randomly fluctuating with amplitude of $\pm 1{ }^{\circ} \mathrm{C}$ over the mean value of $286.7^{\circ} \mathrm{C}$.

b.2 Synchronized fluctuation of inlet coolant flow in all the four coolant loops. The inlet coolant flow is randomly fluctuating with amplitude of $\pm 1 \%$ over the relative flow (100\%).

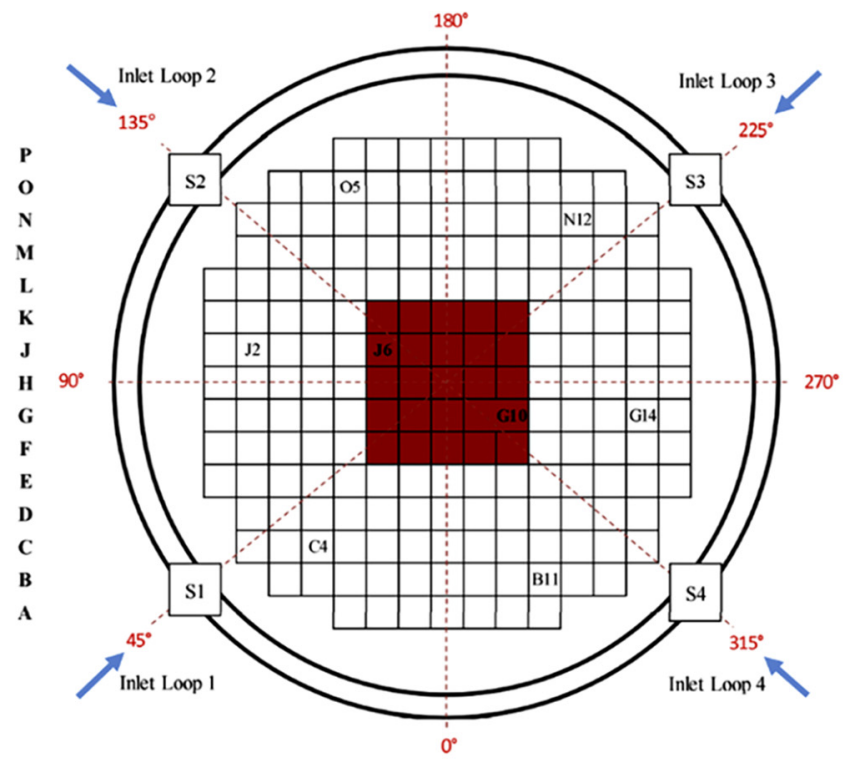

$\begin{array}{llllllllllllllll}1 & 2 & 3 & 4 & 5 & 6 & 7 & 8 & 9 & 10 & 11 & 12 & 13 & 14 & 15\end{array}$

\section{Results from the simulated scenarios}

This section presents the simulation results related to the scenarios described in Section 5.

The simulation results are focused on different aspects:

- The intensity of the different neutron detectors response depending on their distance from the perturbation source.

- The frequency content of the detectors' signals based on the analysis of the APSD.

- The coherence and phase difference between different pair of detectors.

- The comparison of the different scenarios in their distribution of the standard deviation and the NRMS.

- The comparison of the different scenarios with real plant data.

It is important to mention that, the neutron detector's signals in KWU-PWRs have very particular spectral characteristics that have not been fully explained. The content of the signals is four orders of magnitude higher below $1 \mathrm{~Hz}$ than that of beyond $1 \mathrm{~Hz}$. Moreover, out-of-phase relationship has been found between detectors located at opposite half's of the core, and almost zero phase difference below $2 \mathrm{~Hz}$ between lower and upper detectors at the same radial position. Last, it is possible to observe a linear phase relationship with a very low slope which indicates that the transport of the phenomenon is very fast (Czibók et al., 2003; Montalvo et al., 2014a,b).

\subsection{Mechanical vibration scenarios}

\subsubsection{Scenario a.1: synchronized vibration of the central cluster of} $5 \times 5$ fuel assemblies

In Fig. 3, the APSDs of the 8 in-core sensors at the axial level 1 (i.e. core-bottom) are presented. On the left side, the APSDs from the signals are plotted and on the right side, the APSDs calculated with an AR model from each signal are presented. For comparison purposes, it is more convenient to use an AR model since the APSD gets smoother. The different detectors are located at different azimuthal distances from the vibrating fuel elements cluster (Fig. 2,

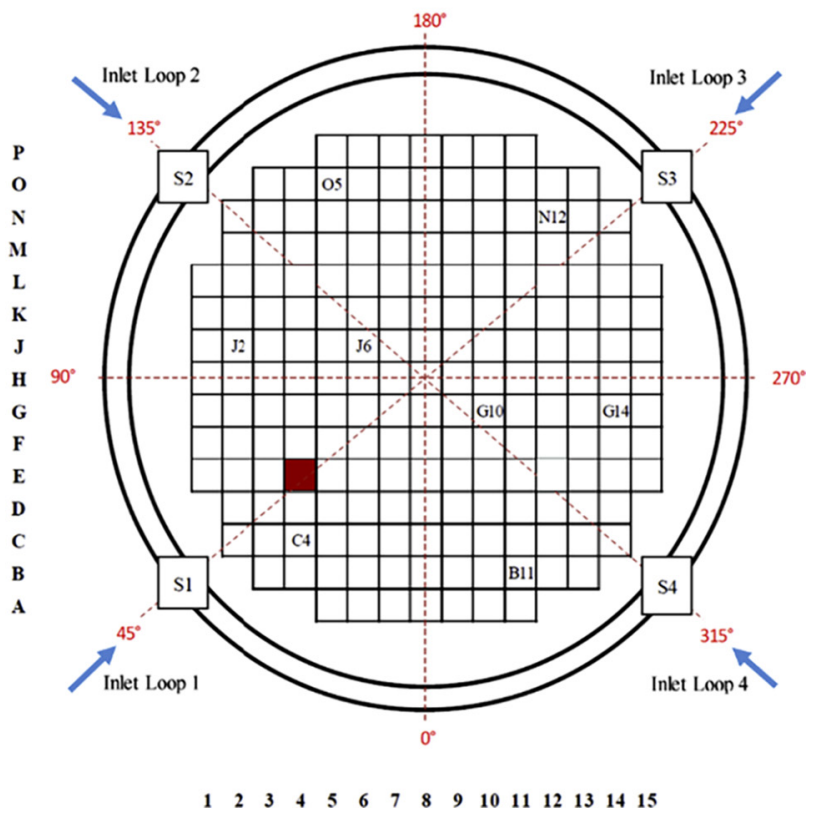

Fig. 2. Radial layout of the sensor strings and fuel elements which are vibrating in scenario a.1 and a.2, respectively. 

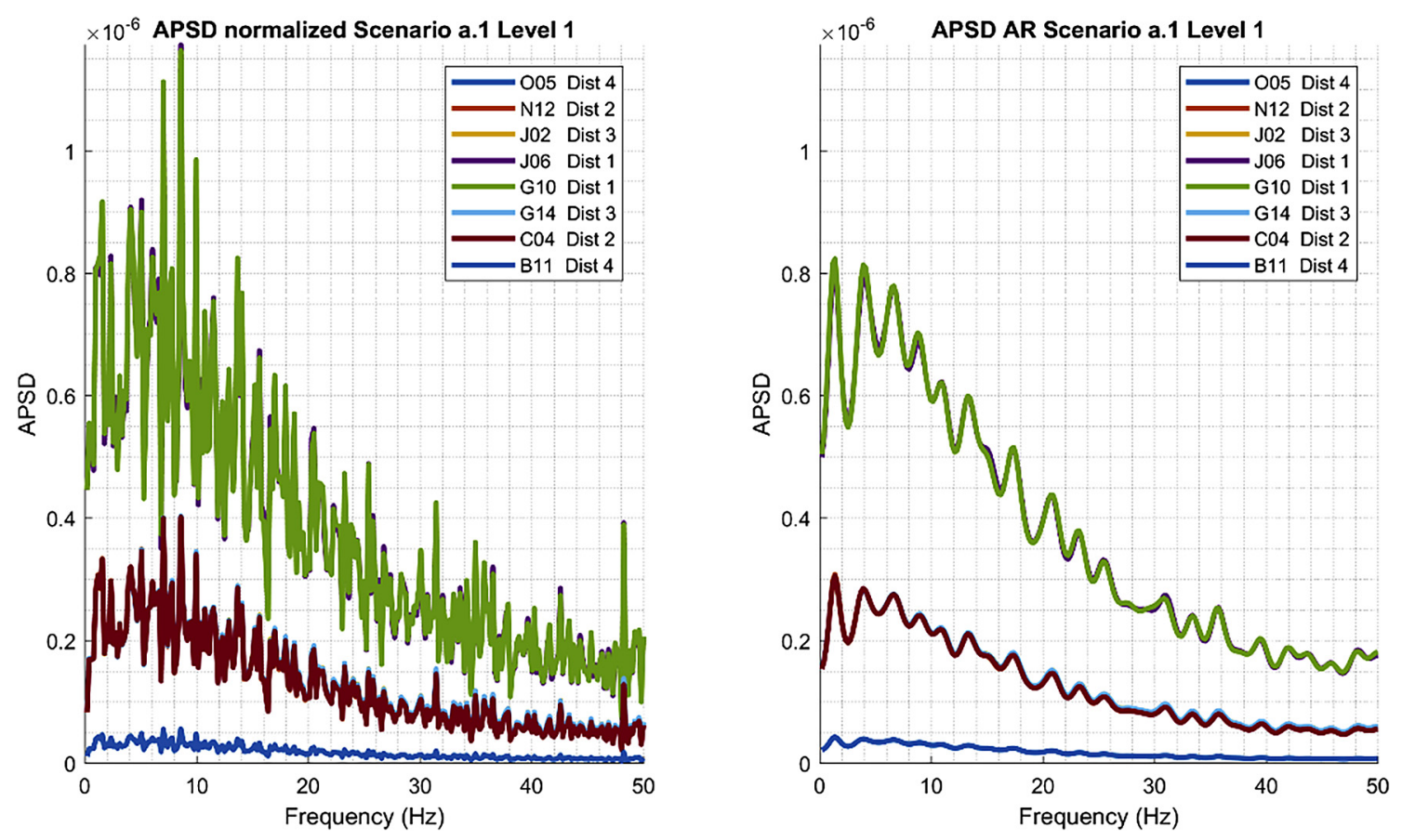

Fig. 3. APSDs of 8 In-core sensors at level 1 (left) and AR model of the same in-core sensors (right).

left plot). These distances are specified in the plot legend. The distances refer to the number of squared nodes apart from the perturbation. It can be observed that the standard deviation decreases with the distance and that the APSDs from detectors at the same distance overlap.

The highest amplitudes of the response are located in the frequency range $0-15 \mathrm{~Hz}$. The lower part of the spectra (i.e. at higher frequencies) does not show high noise amplitude, as it is found in real plant data.

It has to be mentioned that the APSDs, presented in this section, have been normalized in order to ensure that the area below the curve is equal to the signal's variance as it is known from the following equation where the inverse Fourier transform is applied to the APSD (Newland, 2012):

$$
\begin{aligned}
C_{x x}(\tau) & =\int_{-\infty}^{\infty} A P S D(f) e^{i 2 \pi f \tau} d f \text { for } \tau=0 \rightarrow C_{x x}(0) \\
& =\int_{-\infty}^{\infty} A P S D(f) d f
\end{aligned}
$$

The autocorrelation function for $\tau=0$ is:

$C_{x x}(\tau=0)=E[x(t) x(t+\tau)]=E\left[x^{2}\right]$

Fig. 4 presents the following coherence and phase relationships:

- On the left plot, coherence and phase difference for all the 8 sensors at the axial level 1 (i.e. core-bottom), taking as reference the string 4 (J6) sensor.

- On the right plot, coherence and phase difference for all the axial levels in string 4 (J6), taking as reference the level 1 (i.e. core-bottom) sensor.

The coherence is equal to one or almost one in all the pairs plotted. Regarding the radial phase relationship, we have a transverse distribution of phase that divides the reactor into two halves clearly differentiated by $\pm 180^{\circ}$; the strings ( $\left.05, \mathrm{~J} 2, \mathrm{~J} 6, \mathrm{C} 4\right)$ on the left, and the strings (N12, G10, G14, B11) to the right. We must highlight the fact that this out-of-phase relationship takes place in the whole frequency range. In addition, the phase relationship is zero between detectors located at the same string (i.e. same azimuthal location).

\subsubsection{Scenario a.2: vibration of fuel assembly E4}

In this scenario we focus on the coherence and radial phase relationships between sensors at the axial level 1 (i.e. corebottom), which are shown in Fig. 5. In this case, the string C04 is used as reference, which is the closest to the perturbation source.

As it is observed, very strong coherence is observed between all the analyzed sensors. In the case of phase relationships, the out-ofphase behaviour cannot be appreciated for the frequency range of interest.

In this type of scenario, when a single fuel element is vibrating, the out-of-phase relationship does not occur while in previous scenario, (clusters of fuel elements) the out-of-phase extends to the low frequency range (i.e. less than $5 \mathrm{~Hz}$ ), in which thermalhydraulic phenomena have a dominant role.

It has to be mentioned that the real data observations are more similar to the scenario a.1; i.e., opposite sensors have an out-ofphase relationship in the entire frequency range (Runkel, 1987), as it is observed in Fig. 6, in which the coherence and phase difference from opposite ex-core neutron detectors in a real KWU-PWR are plotted.

\subsection{Thermal-hydraulic oscillations scenarios}

In the thermal-hydraulic oscillations scenarios b.1 and b.2, the analysis is focused on observing the coherence and axial phase difference relationships for the six sensors of the string 4 (J06). It is observed in Fig. 7 the scenario b.1 fluctuation of the inlet temperature in $\pm 1{ }^{\circ} \mathrm{C}$ and in Fig. 8 the scenario b.2 fluctuation of the input flow by $1 \%$.

As observed in Figs. 7 and 8 for both scenarios, the coherence is decreasing as the distance from the reference sensor increases, and additionally, all the sensors are in phase above $5 \mathrm{~Hz}$.

In this type of scenarios, it is possible to identify, at low frequencies, the characteristic linear phase that indicates the presence of a transport phenomenon. This is not observed in the previous scenarios of fuel elements vibration. The slope of the lin- 

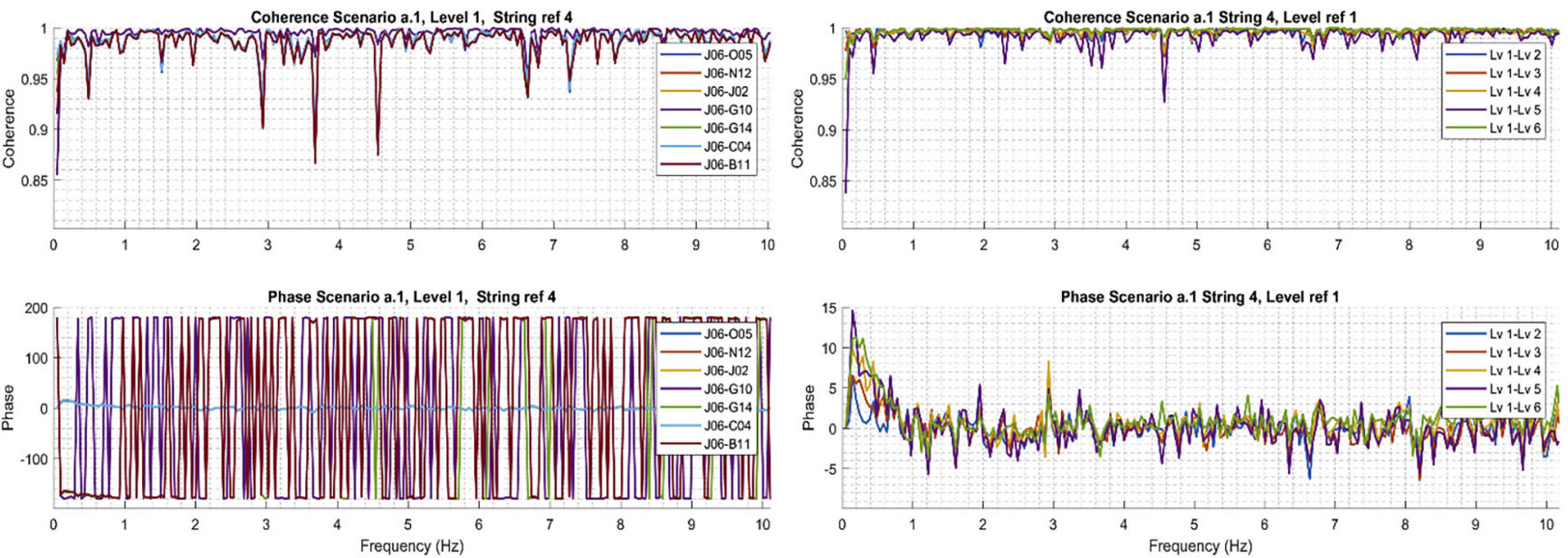

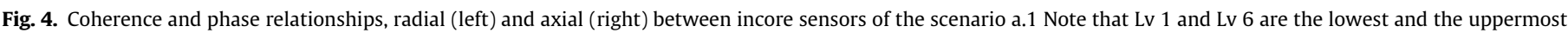
level respectively.
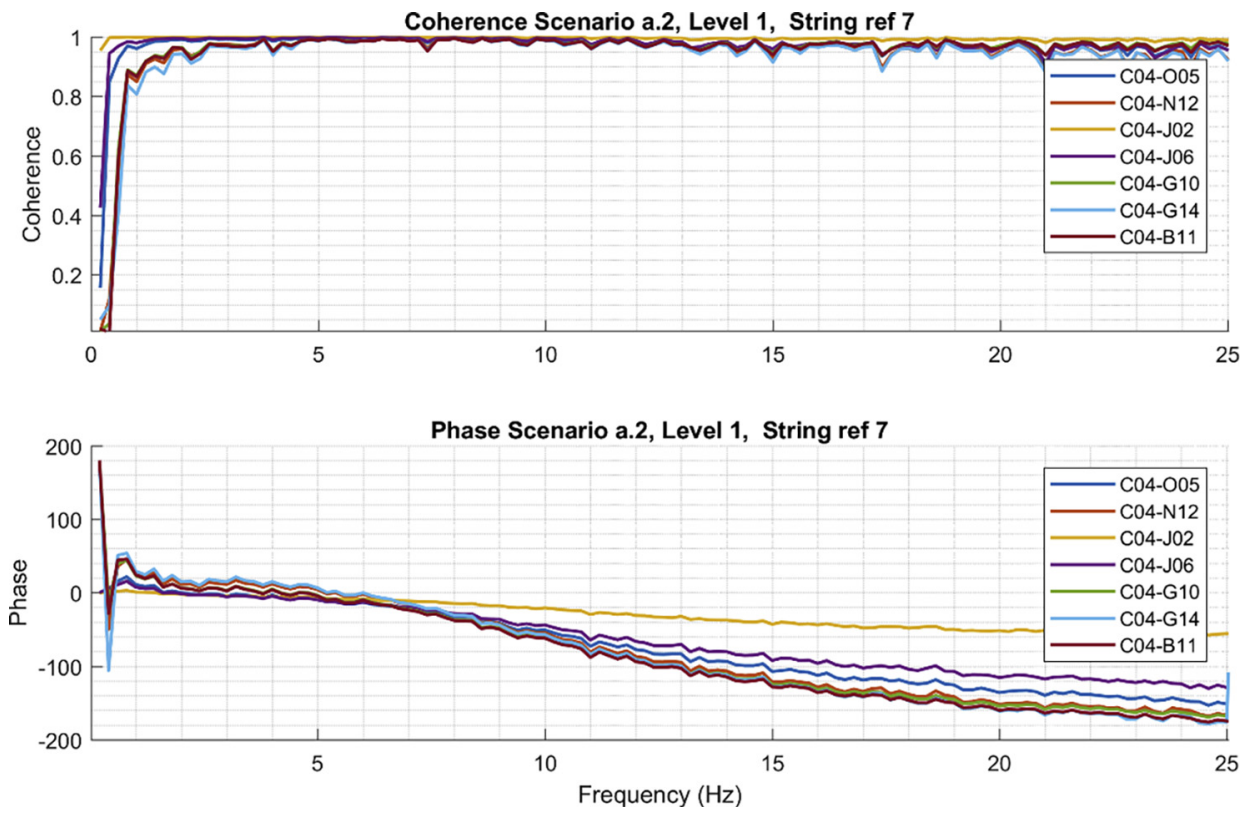

Fig. 5. Coherence and radial phase relationships between incore sensors at level 1 (the lowest level) with string C4 as reference.

ear phase difference is proportional to the transit time according to (Czibók et al., 2003; Runkel, 1987). This means that the higher the slope, the higher the transit time and therefore, the phenomenon of transportation is very slow. Temperature phenomena are characterized as slow processes (from the point of view of dynamics), indicating their big inertia. In this sense, we can see in Fig. 7 that the slope of the linear phase in the low frequency range is much higher than the one in Fig. 8. This indicates that the flow phenomena are transported much faster than the temperature phenomena. In fact, the phase difference between in-core detectors at different axial positions in real plant data show a linear phase with a very low slope (Bermejo, 2015). Keeping in mind this, the flow perturbations scenarios show more similarities with real plant data than the ones with temperature perturbations.

\subsection{Standard deviation and NRMS of neutron noise}

In this subsection, a comparison of the different scenarios based on the values of the signals in terms of standard deviation and NRMS is presented. The standard deviation and NRMS are plotted versus the axial level for two different strings, J02 and J06 (near the edge and in the centre of the core, respectively), and for three simulated scenarios; a.1, central cluster vibration, b.1 and b.2 corresponding to oscillations of coolant inlet temperature and inlet coolant flow rate, respectively.

The standard deviation and NRMS of neutron noise for neutron detector strings J02 and J06 are presented in Figs. 9 and 10, respectively. It is observed in both cases that the standard deviation due to the temperature oscillations is the highest of the three cases, followed by the flow fluctuation scenario, and last the one due to vibrations of the fuel elements.

It is observed that, the profile of the standard deviation and the NRMS in both strings are similar. The NRMS increases with height for inlet coolant flow fluctuations, whereas, the NRMS is decreasing with height for inlet coolant temperature fluctuations, as we can see in Figs. 9 and 10 right plots. According to (Bermejo et al., 2017) temperature fluctuations are supposed to be higher at the bottom since the flow coming from the different loops is not homogenized. In the case of coolant temperature, the exchange of heat along the core reduces fluctuations and makes the differ- 

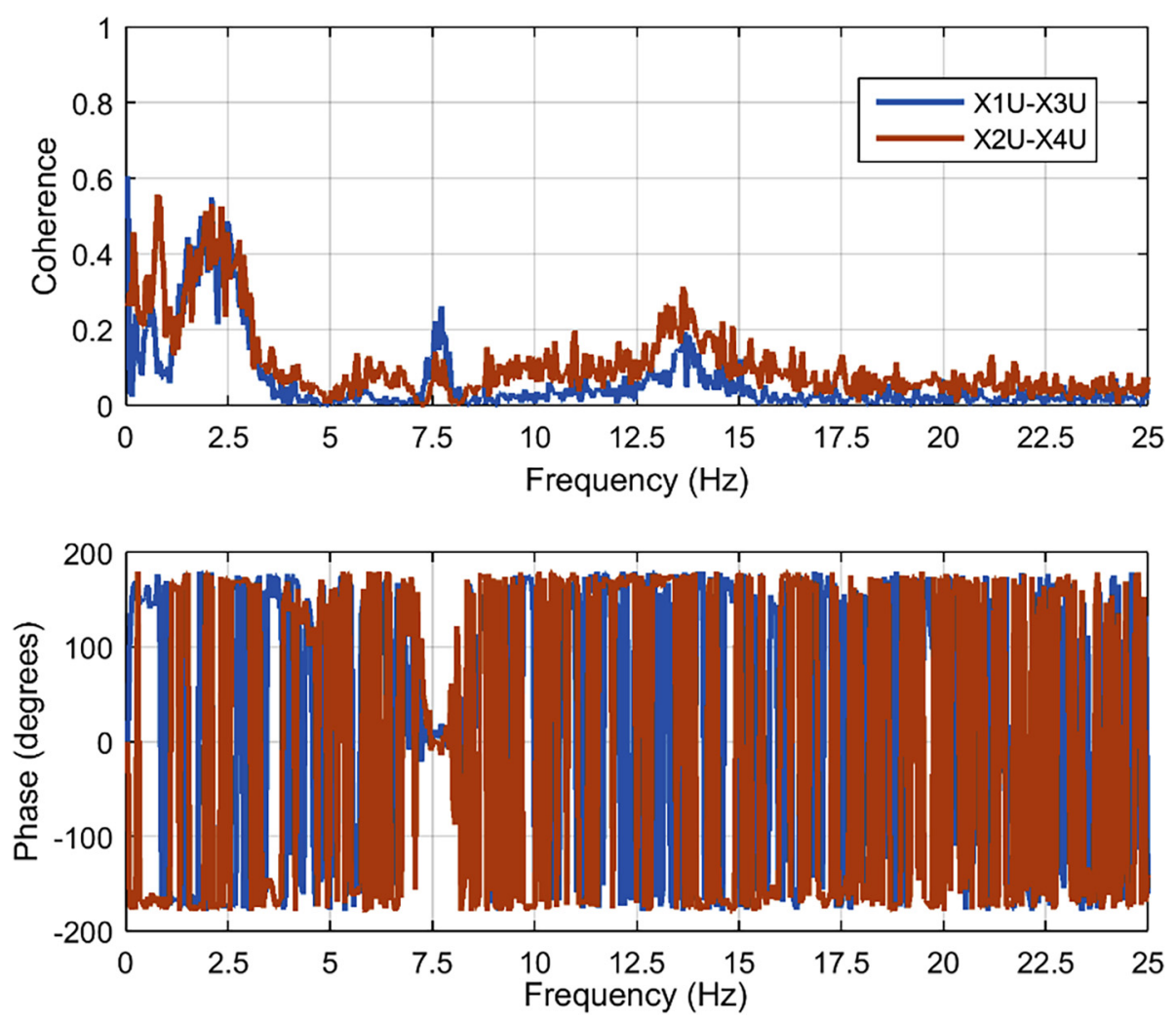

Fig. 6. Coherence and radial phase relationships between ex-core opposite detectors of a KWU-PWR plant.
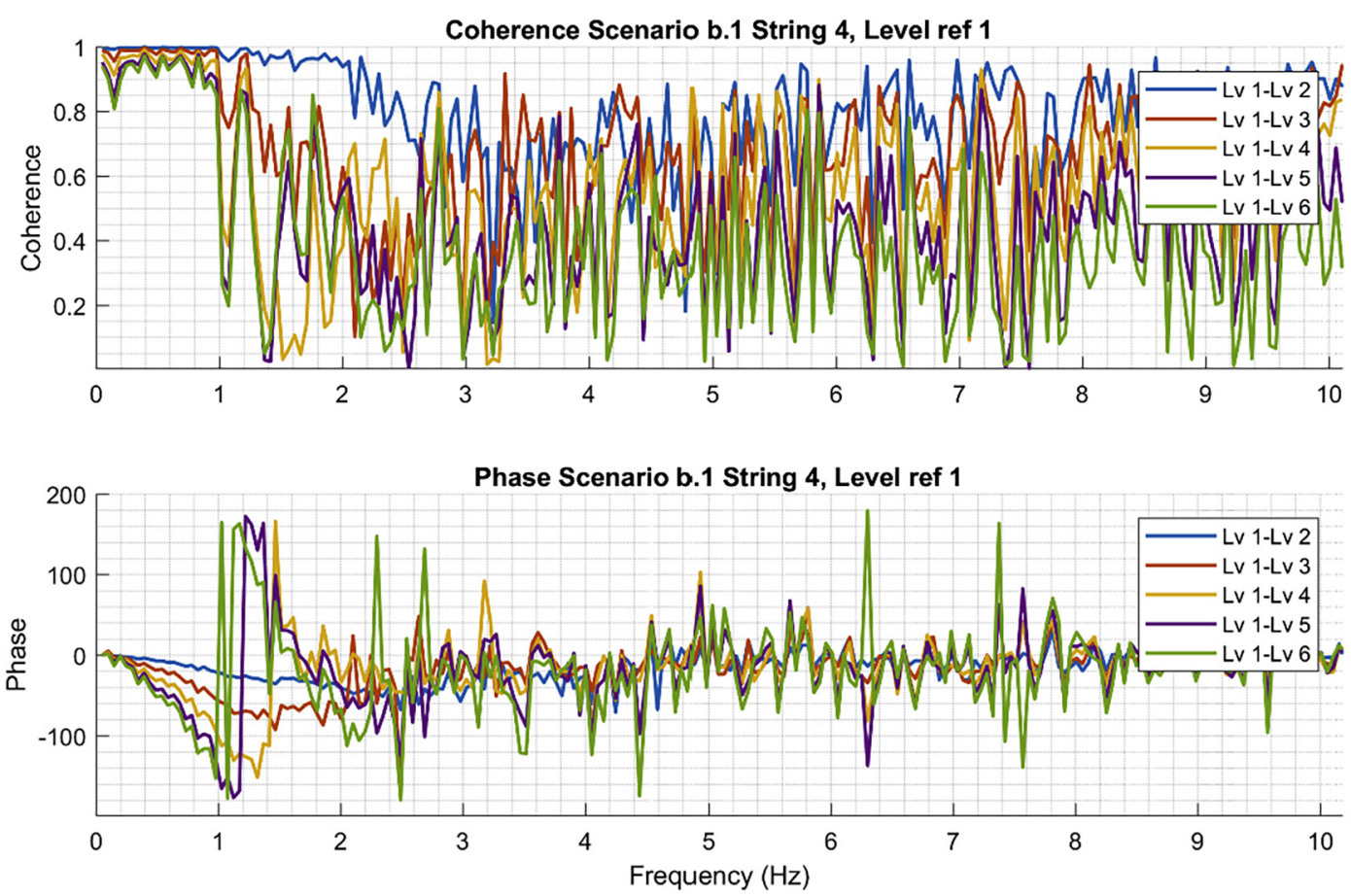

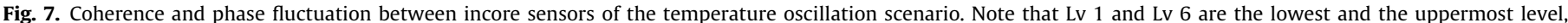
respectively.

ences to homogenize. In the case of inlet flow fluctuations, the nucleate boiling causes a slight increase in the flow fluctuations and consequently a higher NRMS. On the other hand, since the flow perturbations are transported very quickly, the NRMS is not higher at the bottom. These axial profiles coincide to a large extent with the profiles observed in reality, as shown in Fig. 11, in which real plant data from a string of an in-core detector from a KWU-PWR is plotted. 


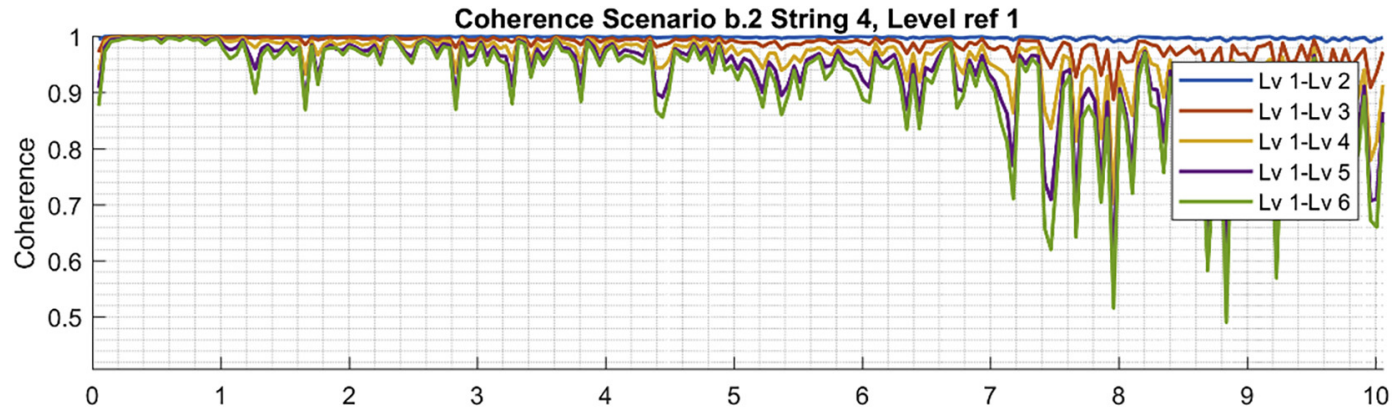

Phase Scenario b.2 String 4, Level ref 1

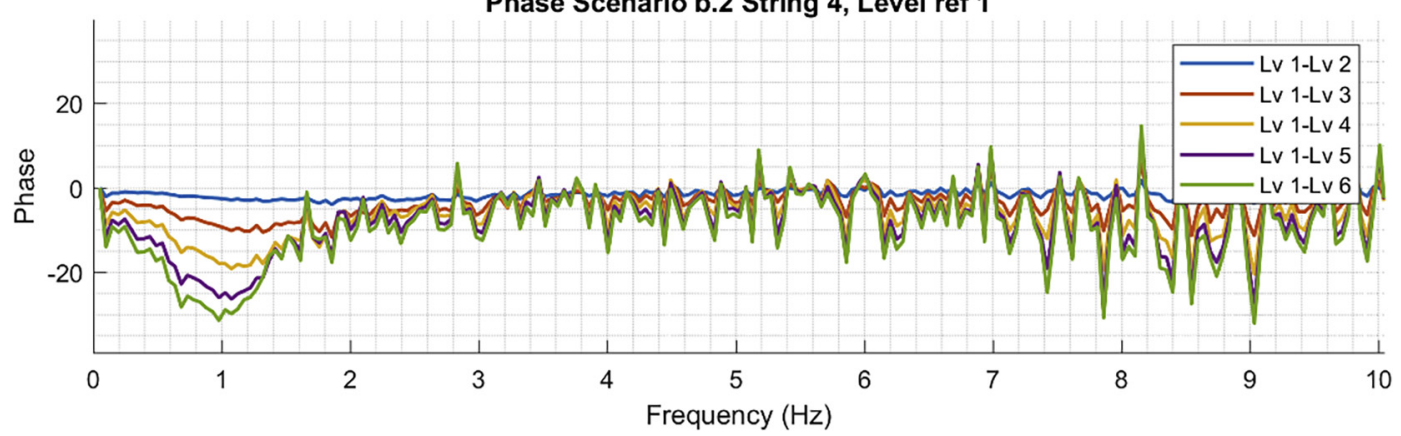

Fig. 8. Coherence and phase fluctuation between incore sensors of flow oscillation scenario. Note that Lv 1 and Lv 6 are the lowest and the uppermost level, respectively.
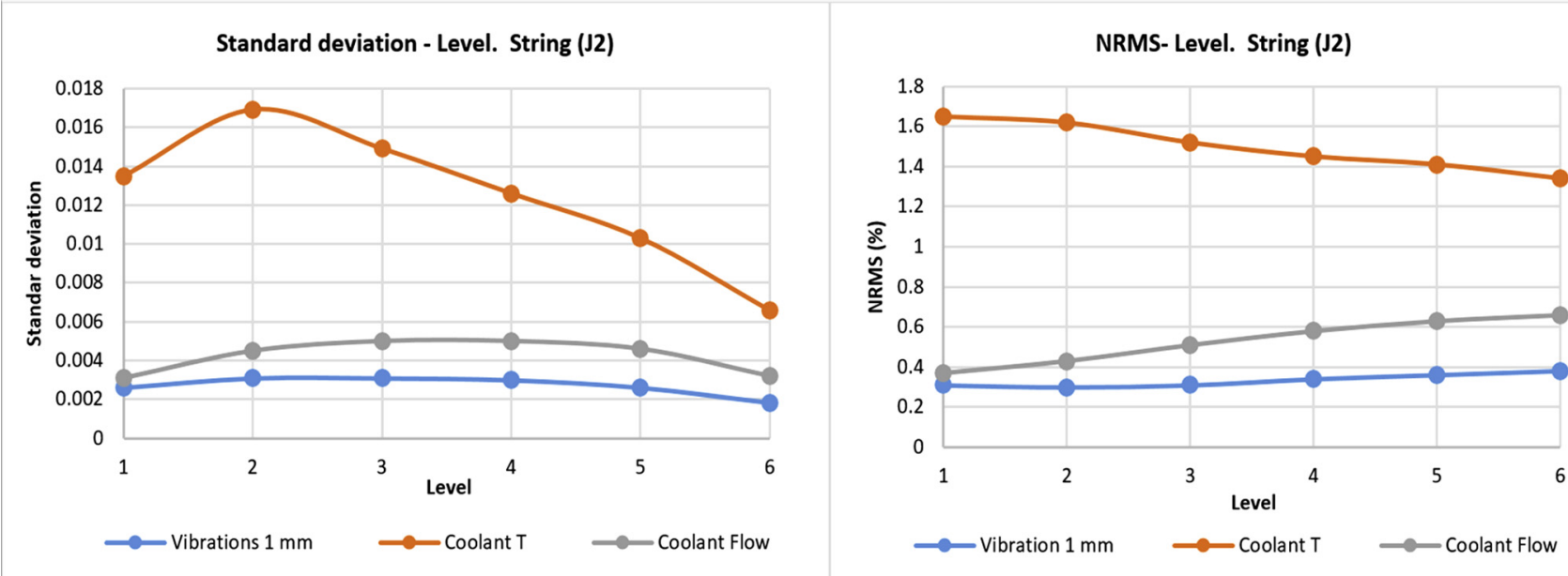

Fig. 9. The standard deviation and NRMS versus the axial level in string J02. Note that Lv 1 and Lv 6 are the lowest and the uppermost level, respectively.
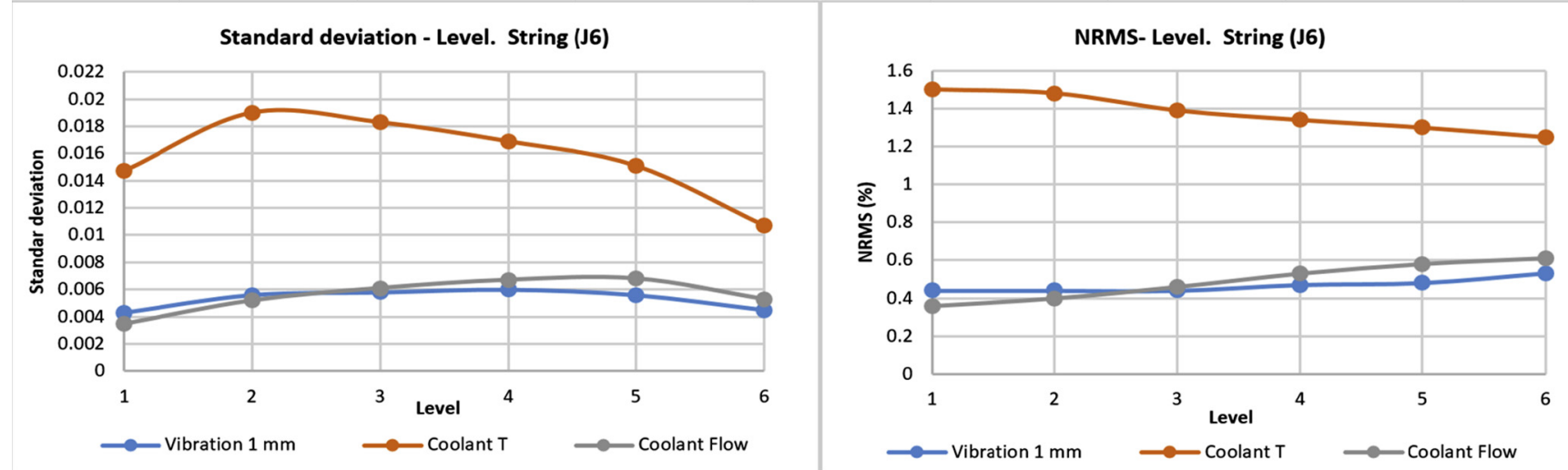

Fig. 10. The standard deviation and NRMS versus the axial level in string J06. Note that Lv 1 and Lv 6 are the lowest and the uppermost level, respectively 


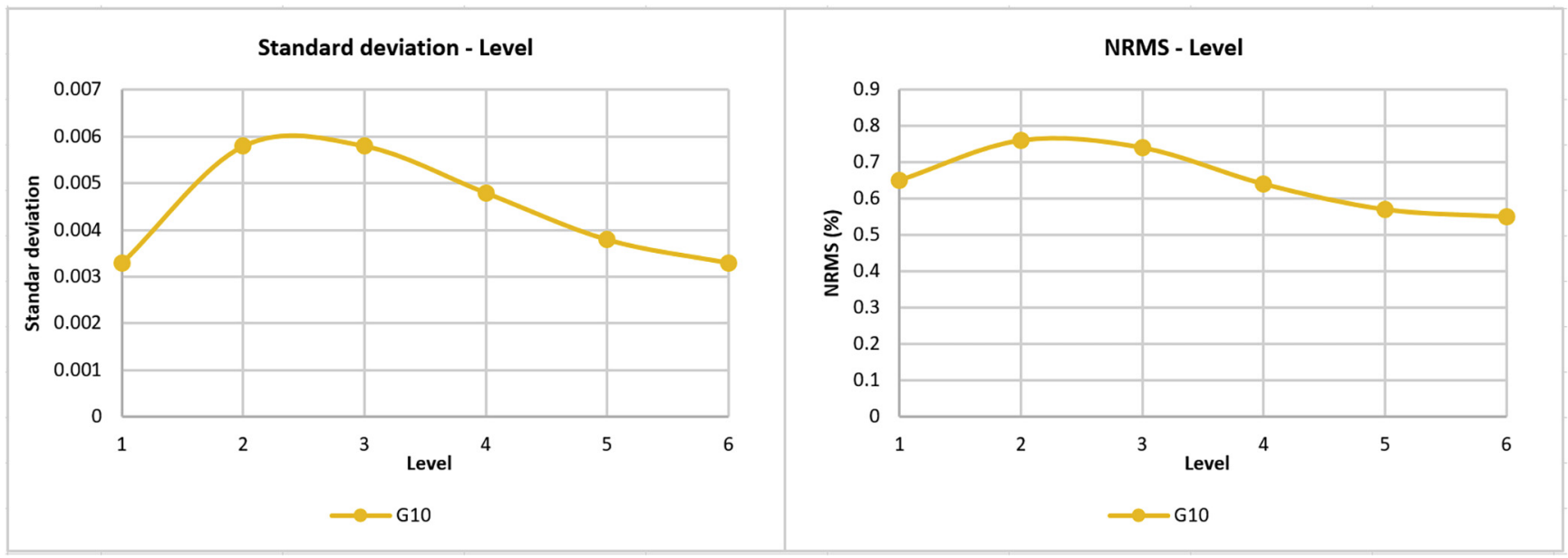

Fig. 11. The standard deviation and NRMS versus the axial level in real data. Note that Lv 1 and Lv 6 are the lowest and the uppermost level respectively.

\section{Discussion}

The three types of simulated scenarios seem to produce separately several spectral characteristics as observed in real neutron noise phenomenology. Three main characteristics observed in the phenomenology of neutron noise and the possible phenomena associated to their causes are discussed in detail in the next sub-sections.

\subsection{High transit speed of the transport phenomenon}

In real data from KWU plants (Bermejo, 2015; Runkel, 1987), the phase difference relationships between in-core sensors of a same string show a very low slope pointing out the existence of a transport phenomenon with a quite high transit speed, as presented in Fig. 12. The real case is similar to what it is observed in the coolant flow oscillation simulated scenarios. Therefore, this characteristic seems to be linked to flow perturbations at the inlet, which unlike the temperature oscillations scenarios, transport along the core almost instantly (see Figs. 7 and 8).

\subsection{Response amplitude at low frequencies, below $1 \mathrm{~Hz}$}

Neutron noise has its highest spectral amplitude around $1 \mathrm{~Hz}$. This characteristic seems to be related to the thermal-hydraulic
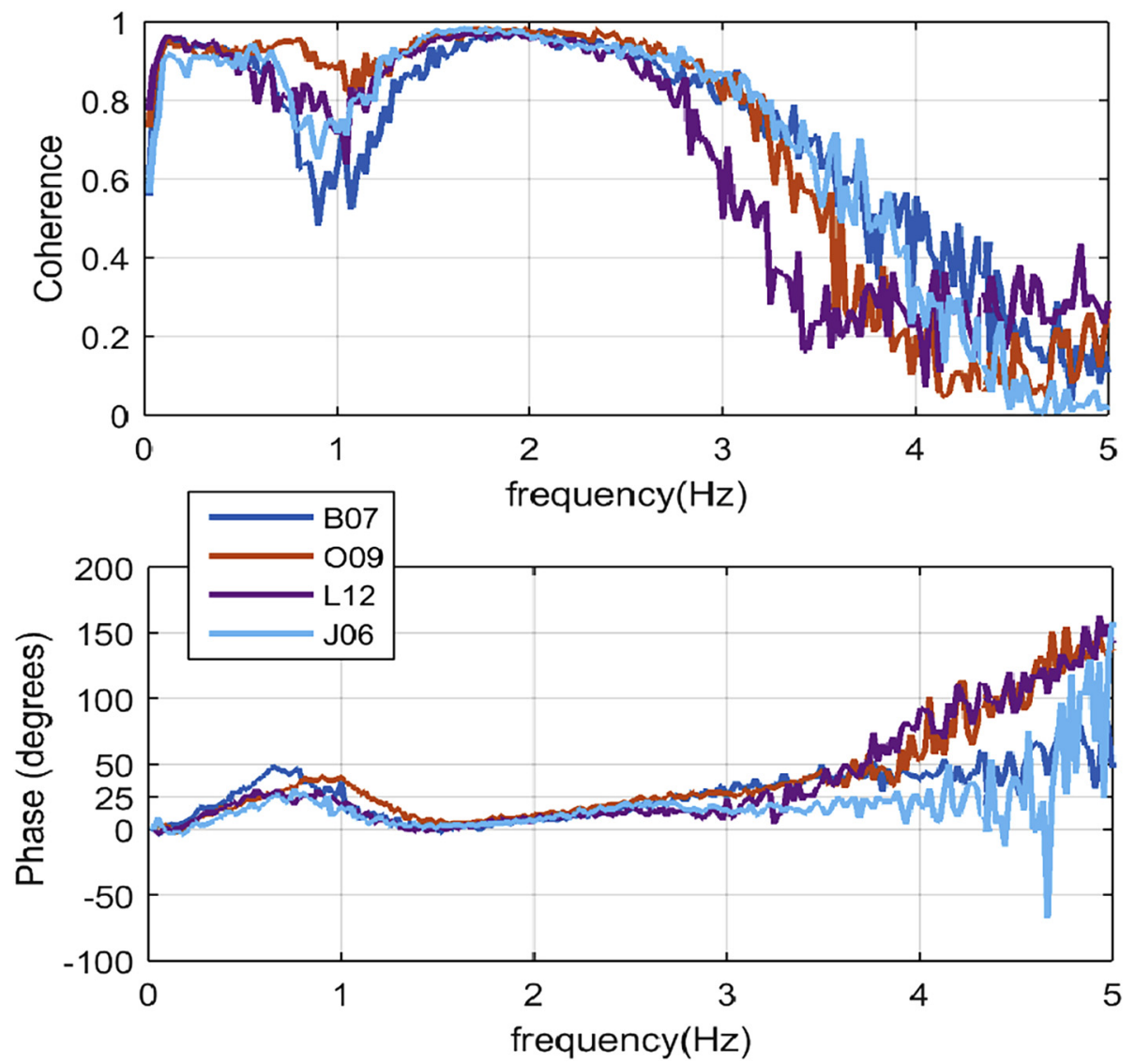

Fig. 12. The phase relationships between Lv 1 and Lv 6 in-core sensors (lower and upper sensors, respectively) of a same string in a KWU-PWR. 

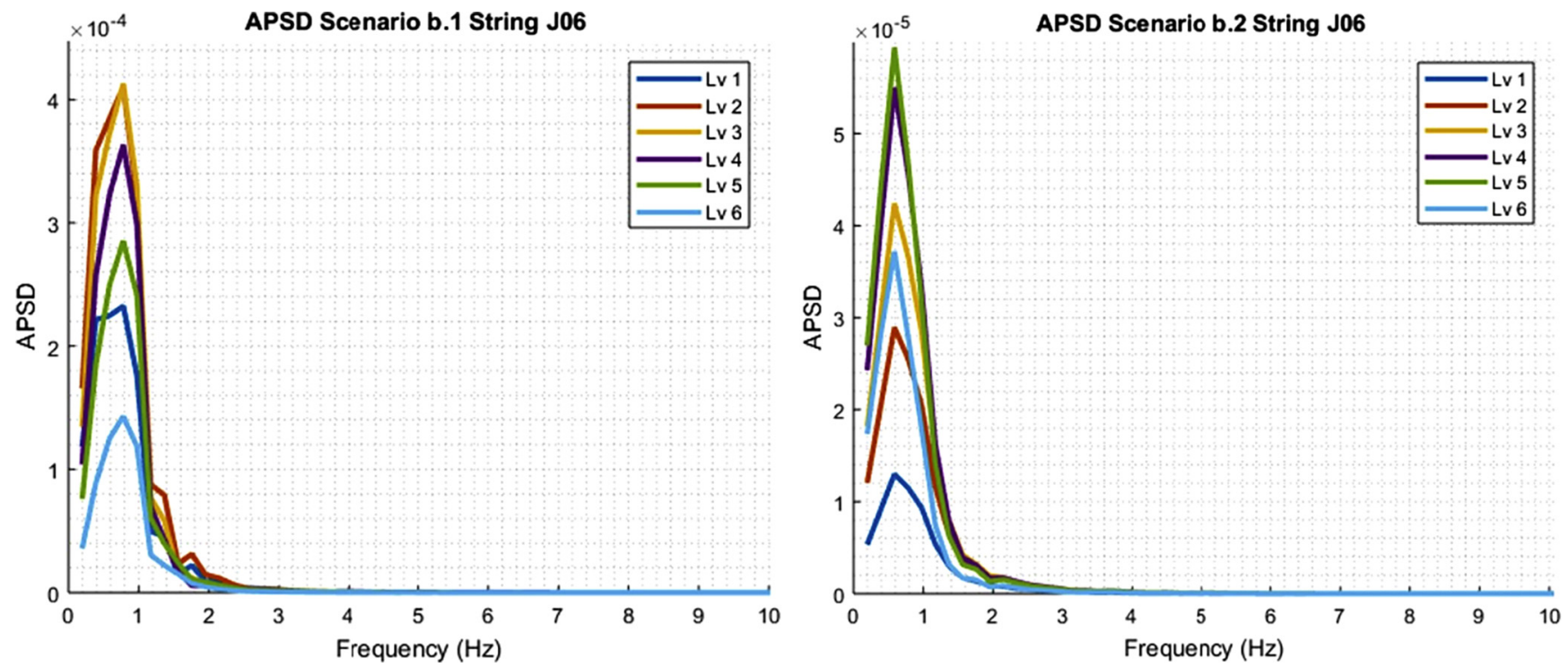

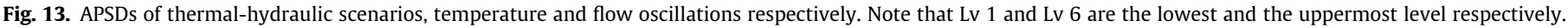

oscillations that are produced in the core and, whose response range is located around $1 \mathrm{~Hz}$, as can be seen in Fig. 13. Temperature and flow oscillations are not directly comparable in a quantitative way. Nevertheless, in this amplitude range, it can be said that the main component of the amplitude seems to be due to temperature oscillations, since this perturbation produces a neutron noise response amplitude four times higher than the flow oscillations and the fuel assemblies' perturbations scenario, as we can also see in Figs. 9 and 10.

Besides, the amplitude of neutron noise depends on the amplitude of the imposed perturbation. For example, if more fuel assemblies are simultaneously vibrating or their vibration displacement is larger, then the noise amplitude due to this mechanism would be enhanced. That can be easily observed by comparing the noise amplitude in the frequency domain between scenarios a1 and a2.

\subsection{Out-of-phase relationship between opposite detectors}

The thermal-hydraulic oscillations simulations are able to justify and reproduce a big part of the spectral characteristics of the neutron noise, however, these scenarios are not enough to try to explain the out-of-phase relationship observed between opposite ex-core sensors in KWU reactors. That has been justified in Fig. 6. For this reason, the mechanical vibrations scenarios are particularly interesting, specifically, the scenario, where a cluster of fuel assemblies is laterally vibrating. This specific scenario leads to an out-of-phase relationship over the entire frequency range, including the $0-15 \mathrm{~Hz}$ band as it is observed in real KWU-PWR data (Runkel, 1987).

The mechanical vibration scenarios with just one fuel assembly do not show the out-of-phase relationship in the low frequency band, where thermal-hydraulic phenomena take place. This fact could suggest that, clusters of fuel assemblies are vibrating in a specific way and causing the characteristic of an out-of-phase relationship in real plants.

\section{Conclusions}

In this paper several simulation results based on the transient nodal code S3K are analysed from the point of view of spectral characteristics of the neutron detector signals. The analysed sce- narios where grouped in two types; on the one hand, scenarios of lateral vibration of fuel assemblies (cluster or single assembly vibration), and on the other hand, thermal-hydraulic parameters fluctuation (inlet coolant temperature and flow).

In the first group of the simulated scenarios (mechanical vibrations), it is observed how the amplitude of the neutron noise decreases rapidly as the distance from the source of perturbation increases. In addition, in-core and ex-core opposite detectors show an out-of-phase relationship in the entire frequency range when clusters of fuel assemblies are vibrating. In the second group of simulated scenarios (thermal-hydraulic perturbations), it is observed a linear phase difference between upper and lower incore detectors located at the same azimuthal location which was found to correspond to a transport phenomenon. The transit times calculated from both scenarios show that the flow perturbations are transported much faster than the temperature ones. Regarding the amplitude of the neutron noise, the temperature oscillations cause the highest response values.

Therefore, in real data, the low transit time observed between upper and lower detectors from the same string can be explained if flow perturbations occur at the core inlet. Nevertheless, the high amplitude of the neutron noise below $1 \mathrm{~Hz}$ seems to be the result of a temperature perturbation.

As seen in the simulations, the spectral characteristics cannot be explained by one single phenomenon. It seems that the amplitude of the neutron noise is due to thermal-hydraulic oscillations and that the characteristic out-of-phase relationship in CPSD between opposite detectors is a consequence of fuel assemblies' vibrations.

The nature of the spectral characteristics observed in the analysis of the neutron noise illustrates the complexity to define the phenomenon and to identify their possible causes, as well as to quantify the relation of each possible cause with the phenomenon itself.

The neutron noise increasing trend is planned to be studied in detail in a future study by using the same simulation methodology as in this paper. To this aim, real core loading patters are going to be simulated. The fuel assemblies which correspond to the latest introduced fuel type design will be simulated to vibrate with the S3K code. The number of vibrating fuel assemblies will follow the increasing number of that specific fuel type which has been 
introduced over the latest cycles in KWU-PWRs. Therefore, the impact of fuel design characteristics on the neutron noise increasing trend will be systematically studied in a follow-up paper.

\section{Acknowledgements}

The research leading to these results has received funding from the Euratom research and training programme 2014-2018 under grant agreement No 754316 (Project CORTEX Core Monitoring Techniques Experimental Validation and Demonstration).

\section{References}

Akaike, H., 1974. A new look at the statistical model identification. Automatic Control, IEEE Trans. 19 (6), 716-723.

Bermejo, J.A., López, A., Ortego, A., 2014. Study of the spatial dependence of the neutron flux oscillations caused by thermohydraulic flucatutions at the core entrance of a PWR reactor. 40th Annual Meeting of the Spanish Nuclear Society.

Bermejo, J., Montalvo, C., Ortego, A., 2017. On the possible effects contributing to neutron noise variations in KWU-PWR reactor: modelling with S3K. Prog. Nucl. Energy 95, 1-7.

Bermejo, J. A. (2015). Noise analysis in PWR (Ph.D. thesis) (in Spanish),

Blázquez, J., Ballestrín, J., 1995. BWR stability boundary surveillance. Prog. Nucl. Energy 29 (3), 147-152.

Chionis, D., Dokhane, A., Belblidia, L., Pecchia, M., Girardin, G., Ferroukhi, H., et al., 2017. SIMULATE-3K analyses of neutron noise response to fuel assembly vibrations and thermal-hydraulics parameters fluctuations. M\&C 2017International Conference on Mathematics \& Computational Methods Applied to Nuclear Science \& Engineering, at Jeju, Korea

Chionis, D., Dokhane, A., Ferroukhi, H., Girardin, G., \& Pautz, A., 2018. Noise phenomenology: Part I - simulation of stochastic phenomena with simulate-3K. PHYSOR 2018: Reactor Physics paving the way towards more efficient systems Cancun, Mexico.

Czibók, T., Kiss, G., Kiss, S., Krinizs, K., Végh, J., 2003. Regular neutron noise diagnostics measurements at the hungarian paks NPP. Prog. Nucl. Energy 43 (1), 67-74.

Dykin, V., Montalvo, C., Nylén, H., \& Pázsit, I., 2014. Ringhals diagnostics and monitoring, final research report 2012-2014 No. CTH-NT-304/RR-19). Gothemburg, Sweden.

Fry, D., March-Leuba, J., \& Sweeney, F., 1984. Use of neutron noise for diagnosis of in-vessel anomalies in light-water reactors No. NUREG/CR-3303; ORNL/TM8774 ON: DE84010431) Oak Ridge National Lab., TN (USA).

Grondey, G., Harms, R., Kumpf, H., Winderl, G., 1991. Low frequency noise in a PWR and its influence on the normal. operational characteristics of the plant. In-core instrumentation and reactor core assessment. Proceedings of Specialist Meeting, In-core instrumentation and reactor core assessment. Proceedings of Specialist Meeting.

Hashemian, H., 2006. Maintenance of Process Instrumentation in Nuclear Power Plants Power Systems. Springer.

Hashemian, H.M., 2011a. On-line monitoring applications in nuclear power plants. Prog. Nucl. Energy 53 (2), 167-181.

Hashemian, H.M., 2011b. Measurement of dynamic Temperatures and Pressures in Nuclear Power Plant Ph.D thesis. The University of Western Ontario.

Hashemian, H., Jiang, J., 2010. A practical review of methods for measuring the dynamic characteristics of industrial pressure transmitters. ISA Trans. 49 (1), $2-$ 9 .
Kostic, L.E.A., 1991. Estimation of MTC in a PWR as Function of Neutron Noise Amplitude. SMORN-6. Gatlinburg, Tennessee, USA.

Kostic, L., Runkel, J., Stegemann, D., 1989. Investigation of the low frequency noise behaviour in a 1350 MW PWR power plant during the first three cycles. Proceeding of IMORN-21 Meeting on Reactor Noise. Villigen, Switzerland.

Kostic, L., Runkel, J., Stegemann, D., 1990. Neutron noise in a power plant near $1 \mathrm{~Hz}$ Proceeding of IMORN-22 Meeting on Reactor Noise. Delft Netherlands.

Kozlowski, T., \& Downar, T.J., 2003. OECD/NEA and US NRC PWR MOX/U02 core transient benchmark No. 2).

Laggiard, E., Runkel, J., 1997. Detection of subcooled boiling in a PWR using noise analysis and calculation of the steam void fraction. Ann. Nucl. Energy 24 (1), 49-54.

Montalvo, C., García-Berrocal, A., Balbás, M., Blázquez, J., 2012. AR modelling and DDS for in situ surveillance of capacitive pressure transmitters. Reactor Knoise Knowledge Transfer Meeting. Prague.

Montalvo, C., Ruiz Zapatero, M., García-Berrocal, A., 2014a. Estimation of the coolant flow velocity in a PWR: three methods based on noise analysis. Annual Meeting of the Spanish Nuclear Society. Valencia.

Montalvo, C. García-Berrocal, A., Bermejo, J., Queral, C., 2014b. Advanced surveillance of resistance temperature detectors in nuclear power plants. Ann. Nucl. Energy 65, 35-40.

Montalvo, C., Pázsit, I., Nylén, H., Dykin, V., 2016. First evidence of the pivotal motion ("tilting mode") of the core barrel in the ringhals-4 PWR. Phys. Reactors. PHYSOR.

Newland, D.E., 2012. An Introduction to Random Vibrations, Spectral and Wavelet Analysis. Dover Publications, Incorporated.

Ortiz-Villafuerte, J., Castillo-Durán, R., Alonso, G., Calleros-Micheland, G., 2006. BWR online monitoring system based on noise analysis. Nucl. Eng. Des. 236 (22), 2394-2404.

Pázsit, I., Montalvo-Martin, C., Tambouratzis, T., \& Dykin, V., 2010. Final report on the research project ringhals diagnostics and monitoring stage 13Chalmers internal report CTH-NT-230/RR-15.

Pázsit, I., Nylén, H., Montalvo-Martin, C., 2014. Refined method for surveillance and diagnostics of the core barrel vibrations of the ringhals PWRs. Proc. PHYSOR, Physor.

Pázsit, I., Montalvo, C., Nylén, H., Andersson, T., Hernández-Solís, A., Cartemo, P.B., 2016. Developments in core-barrel motion monitoring and applications to the ringhals PWR units. Nucl. Sci. Eng. 182 (2), 213-227.

RSK/ESK., 2013. PWR neutron flux oscillations. Statement (457th meeting on 11.04.2013).

Rubiolo, P.R., Young, M.Y., 2008. Characterization of unsteady core flow patterns through noise analysis techniques. J. Power Energy Syst. 2 (1), 435-446.

Runkel, J., 1987. Noise analysis in pressurized water reactor (Ph.D thesis).

Seidl, M., Kosowski, K., Schüler, U., Belblidia, L., 2015. Review of the historic neutron noise behavior in german KWU built PWRs. Prog. Nucl. Energy 85, 668-675.

Spanish Nuclear Safety Council., 2011. Technical judgement proposal. Application of 50 revision of the technical especification of operation of trillo NPP (in spanish) No. CSN/PDT/CNTRI/TRI/1012/129)

Stegemann, D., Runkel, J., 1996. Experience with vibration monitoring in german PWRs obrigheim, grohnde, brokdorf and emsland. Smorn Vii. V, 1.

Thie, J.A., 1981. Power Reactor Noise. American Nuclear Society, La Grange Park, IL, USA.

Tran, H., Pázsit, I., Nylén, H., 2015. Investigation of the ex-core noise induced by fuel assembly vibrations in the ringhals-3 PWR. Ann. Nucl. Energy 80, 434-446.

Viebach, M., Bernt, N., Lange, C., Hennig, D., Hurtado, A., 2018. On the influence of dynamical fuel assembly deflections on the neutron noise level. Prog. Nucl. Energy 104, 32-46.

Zylbersztejn, F., Tran, H.N., Pázsit, I., Demazière, C., Nylén, H., 2013. On the dependence of the noise amplitude on the correlation length of inlet temperature fluctuations in PWRs. Ann. Nucl. Energy 57, 134-141. 